\title{
Towards a Conceptual Framework Fostering Process Comprehension in Healthcare
}

\author{
Michael Zimoch ${ }^{1}$, Rüdiger Pryss ${ }^{1}$, Thomas Probst ${ }^{1}$, Winfried Schlee ${ }^{2}$, Manfred Reichert ${ }^{1}$ \\ ${ }^{1}$ Institute of Databases and Information Systems, Ulm University, Germany \\ 2 Department of Psychiatry and Psychotherapy, Regensburg University, Germany \\ \{michael.zimoch, ruediger.pryss, thomas.probst, manfred.reichert\}@uni-ulm.de \\ winfried.schlee@googlemail.com
}

\begin{abstract}
Despite the widespread use of process models in healthcare organizations, there are many unresolved issues regarding the reading and comprehension of these models by domain experts. This is aggravated by the fact that there exists a plethora of process modeling languages for the graphical documentation of processes, which are often not used consistently for various reasons. Hence, the identification of those factors fostering the comprehension of process models becomes crucial. We have developed a conceptual framework incorporating measurements and theories from cognitive neuroscience and psychology to unravel factors fostering the comprehension of process models within organizations. We believe that a better comprehension of process models will enhance the support of healthcare processes significantly.
\end{abstract}

Keywords-Process model comprehension, conceptual framework, healthcare processes

\section{INTRODUCTION}

Healthcare professionals are facing numerous challenges as they are involved in complex processes everyday [4]. As a consequence, any IT support for healthcare scenarios constitutes a complex endeavour. To capture respective scenarios, process models serve as a major artifact in modern healthcare information systems [1]. Usually, healthcare processes are specified in terms of different modeling languages (e.g., BPMN [5], Petri Nets [6], Flow Charts [7], or IDEF0 [8]), which aggravates model comprehension significantly.

Generally, a process model accumulates tasks, decisions, and resources related to a particular process, i.e., it provides abstractions of procedures and is represented as text or graphical diagram. Besides the creation of process models, their understanding and correct interpretation (i.e., process model comprehension) is of utmost importance to introduce process-awareness in healthcare. Research in this field has shown that the use of graphical process models is beneficial when analyzing and optimizing processes [2]. However, only few experimental settings focus on the factors fostering process model comprehension. To be more precise, cognitive aspects are less understood in this context [3].

Fig. 1 exemplarily presents the results from an eye tracking experiment, illustrating that many factors can be evaluated with respect to process model comprehension. Taking these factors into account and to efficiently compare results of different experiments, we have developed a conceptual framework focusing on the comprehension of process models by adopting concepts from cognitive neuroscience and psychology.

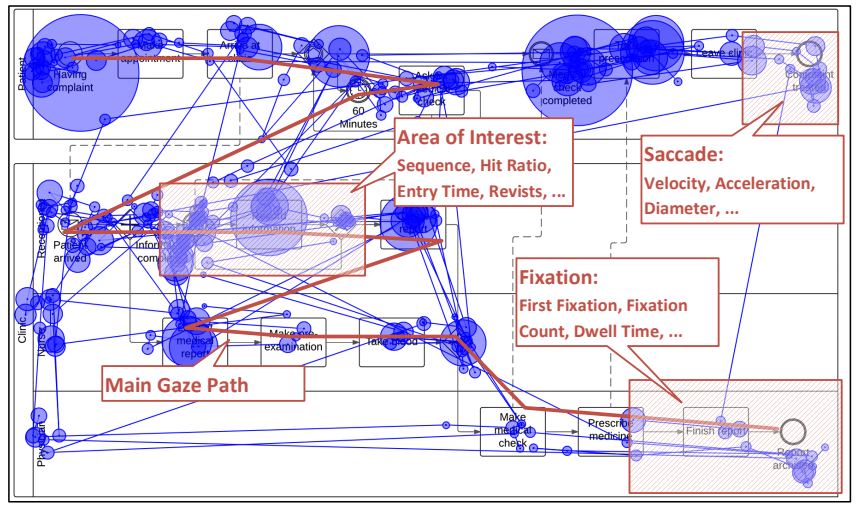

Figure 1: Evaluation of Eye Tracking Results

\section{Conceptual Framework}

By applying cognitive neuroscience and psychology, the approach focuses on the cognitive processes of individuals when reading and understanding process models. We analyze how this information is processed by individuals (e.g., physicians, nurses) in order (1) to improve existing process models as well as (2) to identify rules fostering their comprehension. The latter is a cognitive process trying to establish relations between available information on objects and events in the long term memory, together with information perceived at the moment from the sensory, working, or short term memory. Concerning process models, individuals must cope with the complexities involved in parsing the relevant syntactic, semantic, and pragmatic information of a modeling language. There exist several measurements and theories, which ease the comprehension of a subject, e.g., Cognitive Load Theory (CLT). Fig. 2 illustrates the conceptual framework and its components:

(1) Reference process models in different notations. For graphically documenting business processes, there exists a variety of process modeling languages. Still, the usage of these languages is not always consistent as standardized modeling procedures do not exist. Therefore, the conceptual framework considers various modeling languages. 


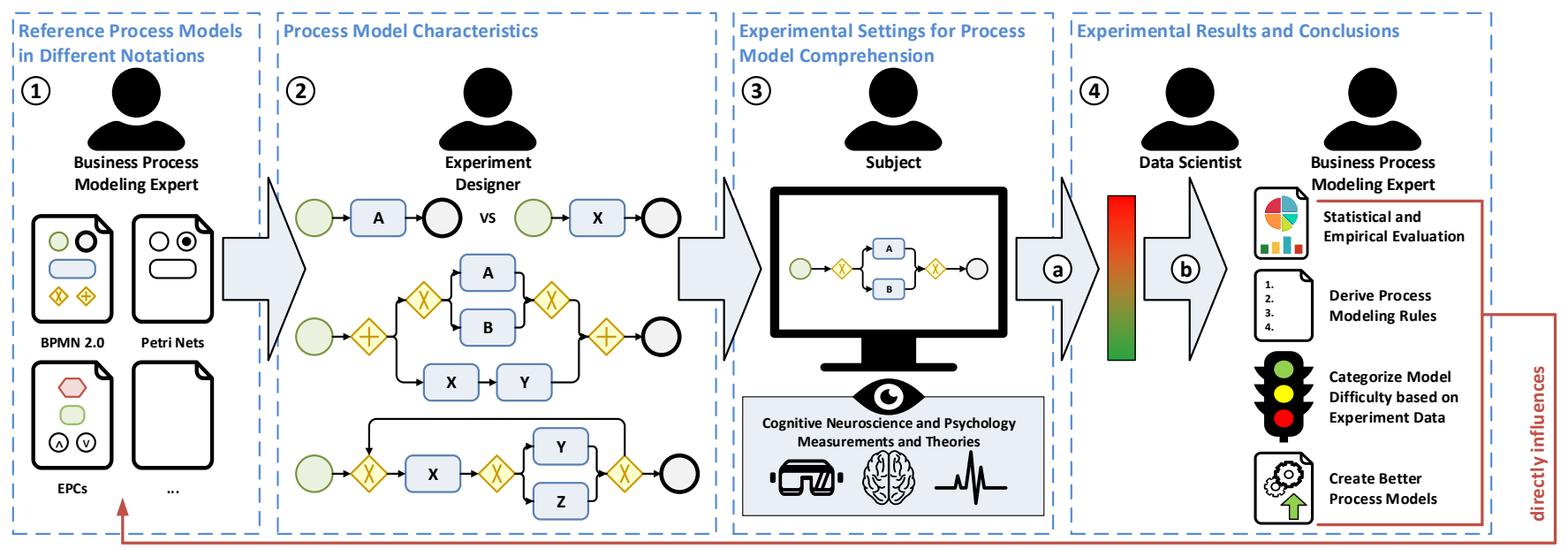

Figure 2: Conceptual Framework

(2) Process model characteristics. There are factors related to process modeling that influence an individual's capability to comprehend process models, e.g., the chosen graphical representation or level of complexity. Such factors must be carefully considered when designing experiments.

(3) Experimental settings for process model comprehension. Individuals perceive graphical representations differently, depending on personal factors (e.g., expertise). Throughout a series of experiments, which make use of concepts from cognitive neuroscience and psychology (e.g., CLT), the identification of stumbling blocks and obstacles as well as aspects fostering process model comprehension will be addressed by the conceptual framework.

(4) Experimental results and conclusions. Findings obtained from the experiments are analyzed by scientists using different methods (e.g., similarity matching). The results are used to rate and classify individuals with respect to process model comprehension (cf. Fig. 1 (a)). This classification reflects the perceived difficulty of an individual regarding process model comprehension and the importance of personal factors. Taking these results into account, further steps can be derived (cf. Fig. 1 (b)). Tab. 1 depicts approaches, for which the conceptual framework has been used so far as well as numbers of subjects and analyzed models.

\begin{tabular}{lcc}
\hline Approaches & No. Subjects & No. Models \\
\hline \hline Construal Level Theory & 136 & 262 \\
Eye Tracking & 36 & 432 \\
Electrodermal Activity & 7 & 112 \\
Heart Rate & 3 & 72 \\
\hline
\end{tabular}

Table I: Experiments using the Conceptual Framework

\section{SUMmARY AND OUTLOOK}

The use of cognitive neuroscience and psychology as basic pillar of the conceptual framework offers promising perspectives with respect to process model comprehension.
The goal is to provide optimized rules enabling a better process model comprehension as well as directives for creating better process models. Currently, we conduct a series of experiments with different concepts originating from cognitive neuroscience and psychology to unravel the factors facilitating process model comprehension.

\section{REFERENCES}

[1] R. Lenz and M. Reichert, IT Support for Healthcare Processes - Premises, Challenges, Perspectives, in Data and Knowledge Engineering, vol 61, 2007, 39-58

[2] A. Ottensooser and A. Fekete and H.A. Reijers and J. Mendling and C. Meicstas, Making Sense of Business Process Descriptions: An Experimental Comparison of Graphical and Textual Notations, in J of Sys and Soft, vol 85, 2012, 596-606

[3] K. Figl and R. Laue, Cognitive Complexity in Business Process Modeling, in Adv Inf Sys Eng, 2011, 452-466

[4] M. Reichert, What BPM Technology Can Do for Healthcare Process Support, in AIME'11, (2011), 2-13

[5] E. Rolón and F. García and F. Ruíz and M. Piattini and L. Calahorra, Luis, Healthcare Process Development with BPMN, in Handbk of Res on Dev in E-Health and Telemedicine: Tech and Soc Pers, IGI Global, 2010, 1024-1047

[6] M. Jansen-Vullers and H. Reijers, Business Process Redesign at a Mental Healthcare Institute: A Coloured Petri Net Approach, in Proc 6th Workshop and Tutorial on Practical Use of Coloured Petri Nets and the CPN Tools, 2005, 21-38

[7] U. Müller and A. Exadaktylos and C. Roeder and M. Pisan and S. Eggli and P. Jüni, Effect of a Flow Chart on Use of Blood Transfusions in Primary Total Hip and Knee Replacement: Prospective Before and After Study, in BMJ, 2004, 934-938

[8] C. Wong and G. Geiger and Y. Derman and C. Busby and M. Carter, Healthcare Process Analysis: Redesigning the Medication Ordering, Dispensing, and Administration Process in an Acute Care Academic Health Sciences Centre, in Proc of the 35th Conf on Win Sim: Driving Innovation, 2003, 1894-1902 